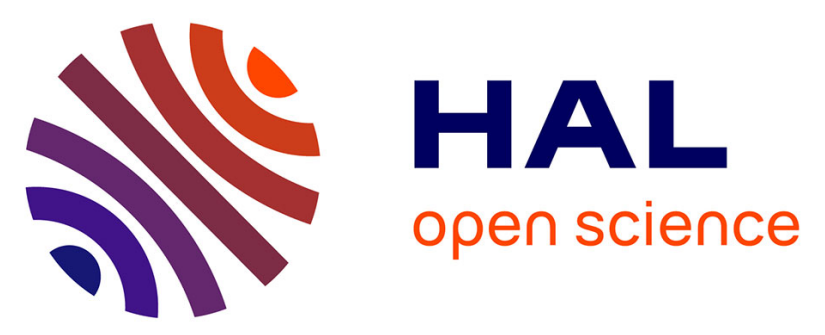

\title{
Injection molding simulation: Taking into account the process history to predict the anisotropy in the end-use properties
}

Luisa Silva, Houssem Miled, Patrice Laure, Thierry Coupez

\section{- To cite this version:}

Luisa Silva, Houssem Miled, Patrice Laure, Thierry Coupez. Injection molding simulation: Taking into account the process history to predict the anisotropy in the end-use properties. Materials Processing and Design, Modeling, Simulation and Applications, NUMIFORM '07: 9th International Conference on Numerical Methods in Industrial Forming Processes, Jun 2007, Porto, Portugal. pp.Pages 355-360, 10.1063/1.2740837 . hal-00510562

HAL Id: hal-00510562

https://hal-mines-paristech.archives-ouvertes.fr/hal-00510562

Submitted on 1 Apr 2011

HAL is a multi-disciplinary open access archive for the deposit and dissemination of scientific research documents, whether they are published or not. The documents may come from teaching and research institutions in France or abroad, or from public or private research centers.
L'archive ouverte pluridisciplinaire HAL, est destinée au dépôt et à la diffusion de documents scientifiques de niveau recherche, publiés ou non, émanant des établissements d'enseignement et de recherche français ou étrangers, des laboratoires publics ou privés. 


\title{
Injection Molding Simulation : Taking Into Account the Process History to Predict the Anisotropy in the End-Use Properties
}

\author{
Luisa Silva, Houssem Miled, Patrice Laure and Thierry Coupez \\ CEMEF - Ecole des Mines de Paris - UMR CNRS 7635, rue Claude Daunesse, 06904 Sophia-Antipolis, France \\ FEUP, Faculdade de Engenharia, Universidade do Porto, Rua Dr Roberto Frias s/n, 4200-465 Porto, Portugal
}

\begin{abstract}
This work's context is an industrial project aiming the accurate modeling of the injection molding process [1]. 3D numerical simulation of the different stages is considered: during processing, anisotropy of the stress state build up affects its mechanical, optical or dimensional properties, and induces warpage once the part is ejected.

A first example of injection molding of reinforced thermoplastics will be treated. In this case, we will consider that during the injection step, an orientation will be induced by the flow. Furthermore, the thermoplastic matrix will pass from the liquid to the solid state, and orientation and stresses will remain frozen. Evolution of orientation or extra stress is computed using the Folgar and Tucker equation, with continuous or discontinuous approximations. Results are obtained in a 3D complex industrial part.
\end{abstract}

Keywords: Polymer Injection molding, Solidification, Flow Induced Orientation, Finite element method.

PACS:

\section{INTRODUCTION}

During injection molding of thermoplastic polymers, the material's answers to the high shear rates by orienting its molecular chains (or the reinforcements) along the main directions of shearing. Orientation affects the physical and mechanical properties, dimensional stability and the aspect of the finished product. It results in anisotropy of mechanical, optical and thermal properties. For example, the anisotropy of the thermal conductivity and the thermal expansion coefficient influences directly the time dependent spatial temperature distribution and subsequently the development of thermal stresses during the entire process. Anisotropy in elastic moduli influences warpage and anisotropy of the refractive index influences birefringence patterns.

Orientation can be seen as the frozen in picture, at a molecular scale, of the deforming material as it existed during processing. From the above description, we conclude that the development of the residual stress field in an injection molded part is rather complex. The flow-induced and thermal-induced stresses are coupled [1] and no single constitutive equation seems capable of describing the full behavior of the polymer through the injection, packing and cooling stages.

\section{FLOW INDUCED ANISOTROPY}

Basically, there are two main sources of residual stresses. Firstly, due to the viscoelastic nature of polymers, normal stresses develop during filling and packing/holding stages, usually these flow-induced stresses are relatively small. However, they give rise to large molecular orientations which affect the mechanical and optical behavior of the part. They also influence differences in the shrinkage behavior in directions perpendicular and parallel to the flow direction. The second cause of residual stresses is the rapid increase in rigidity of the material as it passes through the glass transition point. Across the molded part thickness, a highly non-uniform temperature distribution exists. Consequently, each material point solidifies at a different time, leading to differential shrinkage causing thermally induced stresses. From experimental and previous simulation work, we are able to understand the mechanism of formation of flow-induced stresses in injection molding, as well as to give a qualitative representation of this stresses:

- during filling, the layer in contact with the cold mold wall solidifies rapidly because its cooling rate is very high, but near the surface the molecules are highly 
oriented due to the high shear rate. In the central layer molecules do not orient since there is no shear rate;

- at the end of filling the molecular orientation relaxes quickly since the flow is very small and the temperature is still high;

- during packing when the temperature becomes low, molecular orientation near the midplane may grow again;

- at the end of cooling very few stresses were relaxed (the relaxation times increase beyond the process scope) and will cause shrinkage and deformation once the part ejected.

\section{Orientation Tensors}

The simplest representation of orientation is to look, at an appropriate scale, to the end-to-end vector $p$ of the entity that is orienting (Figure 1). For example, let us consider that a macromolecular chain can be represented by $N$ monomers for which we define the end-to-end vector $p$. If we call $\varphi(p)$ the probability

distribution function to find $p$, then the following evolution law for the distribution of probability can be established:

$$
\frac{\partial \varphi}{\partial t}=-\nabla \cdot\left[\varphi \frac{\partial \underline{p}}{\partial t}\right]+D \frac{\partial^{2} \varphi}{\partial \underline{p}^{2}}
$$

where $D$ is a Brownian diffusion coefficient Nevertheless, function $\varphi(\underline{p})$ is not often known Actually, the second order tensor $\underline{\underline{a}}$ is the most used to describe at the macroscopic (process) scale the orientation state, even if it may include a loss of information. This tensor represents the spatial mean of the tensorial product of $p$ :

$$
\underline{a}=\int(\underline{p} \otimes \underline{p}) \varphi(\underline{p}) d p=\langle\underline{p} \otimes \underline{p}\rangle
$$

$\underline{\underline{a}}$ is a symmetric tensor at unitary trace. The general equation for the evolution of $\underline{\underline{a}}$ [2] is:

$$
\frac{\partial \underline{\underline{a}}}{\partial t}+\underline{v} \cdot \nabla \underline{\underline{a}}+\underline{\underline{B}} 1 \cdot \underline{\underline{a}}+\underline{\underline{a}} \cdot \underline{\underline{B}}=2\left(\underline{\underline{a}}+\underline{\underline{a}}_{4}: \underline{\underline{v}}\right)+\frac{1}{\theta}\left(\underline{\underline{a}}-\frac{\underline{\underline{1}}}{3}\right)=0
$$

on $\Omega \times] 0, \tau[$, where $\underline{v}$ is the macroscopic velocity vector.
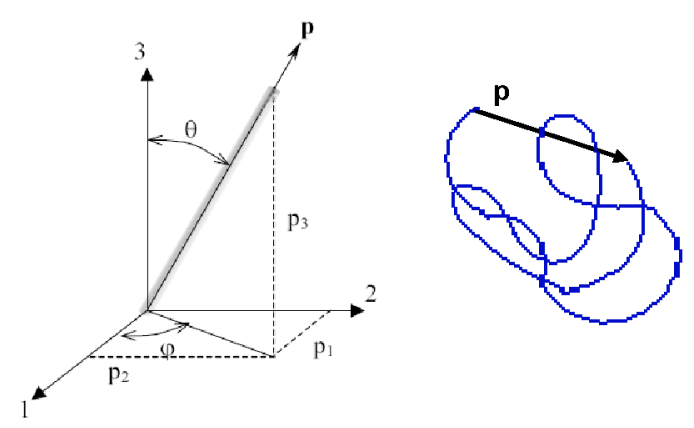

FIGURE 1. Representation of a fiber or a chain through its end-to-end vector[3].

This equation is valid for both fiber and chain orientation :

- Macromolecular chains: $B_{1}=-\nabla \underline{v}$ and $B_{2}=-\nabla \underline{\underline{v}}^{t}$;

- Fiber orientation: $B_{1}=-\lambda \underline{\underline{D}}(\underline{v})+\underline{\underline{Q}}(\underline{v}) \quad$ and $B_{2}=\lambda \underline{\underline{D}}(\underline{v})+\underline{\underline{O}}(\underline{v})$, where $\lambda$ is a function of the fiber shape factor, $\underline{\underline{O}}(\underline{v})$ is the rotation tensor and $\underline{\underline{D}}(\underline{v})$ is the deformation rate tensor, given by :

$$
\underline{\underline{D}}(\underline{v})=\frac{1}{2}(\nabla \underline{v}+\nabla \underline{v}) \text { and } \underline{\underline{O}}(\underline{v})=\frac{1}{2}\left(\nabla \underline{v}-\nabla \underline{v}^{f}\right)
$$

In equation (3), there is the fourth order orientation tensor, given by :

$$
\underline{\underline{a}}_{4}=\langle\underline{p} \otimes \underline{p} \otimes \underline{p} \otimes \underline{p}\rangle
$$

To relate this tensor with the second order one, we use a closure approximation. The most one is the quadratic:

$$
\begin{aligned}
& \underline{\underline{a}}_{4}=\langle\underline{p} \otimes \underline{p} \otimes \underline{p} \otimes \underline{p}\rangle \approx\langle\underline{p} \otimes \underline{p}\rangle \otimes\langle\underline{p} \otimes \underline{p}\rangle \\
& \Rightarrow \underline{\underline{a}}_{4} \approx \underline{\underline{a}} \otimes \underline{\underline{a}}
\end{aligned}
$$

The term in $\underline{\underline{a}}_{4}$ in the orientation equation will be approached by $2\left(\underline{\underline{a}}_{4}: \nabla \underline{v}\right) \approx 2(\underline{\underline{a}}: \nabla \underline{v}) \underline{\underline{a}}$, and the orientation evolution equation is finally

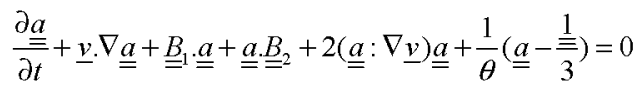

Knowing the eigenvalues and the eigenvectors of the orientation tensor, this last one can be described by an ellipsoid in 3D or by an ellipsis in $2 \mathrm{D}$ - the 
eigenvectors define the direction along each axis, while the eigenvalues represent their lengths. Figure 2 shows some examples of values of this tensor, for some orientation tensors, in particular for the isotropic state.

\section{NUMERICAL RESOLUTION OF TE ORIENTATION EQUATION}

\section{Standard Galerkin}

Let us consider an implicit Euler discretization in time. Being the orientation equation non-linear due to the closure approximation, we use the following linearization: $\quad 2(\underline{\underline{a}}: \nabla \underline{v}) \underline{\underline{a}} \approx 2\left(\underline{\underline{a}}^{t-\Delta t}: \nabla \underline{\underline{v}}\right) \underline{\underline{a}}^{t} . \quad$ The orientation equation is then:

$$
\frac{\partial \underline{\underline{a^{t}}}}{\partial t}+\underline{v} \cdot \nabla \underline{\underline{a^{t}}}+\underline{\underline{B}} \cdot \cdot \underline{\underline{\underline{a}}}+\underline{\underline{a}}^{t} \cdot \underline{\underline{B}}_{2}+2\left(\underline{\underline{a}}^{t-\Delta t}: \nabla \underline{v}\right) \underline{\underline{a^{t}}}+\frac{1}{\theta}\left(\underline{\underline{a^{t}}}-\frac{\underline{\underline{\underline{1}}}}{3}\right)=0
$$

If we consider the bilinear forms

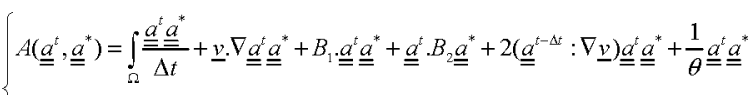

$$
\begin{aligned}
& l(\underline{\underline{\underline{a}}})=\int_{\Omega} \frac{\underline{\underline{a^{t}}} \underline{\underline{\underline{\Delta}}}: \underline{\underline{\underline{a}}}^{*}}{\Delta t}+\frac{\operatorname{tr}\left(\underline{\underline{\underline{a^{*}}}}\right)}{3}
\end{aligned}
$$

The problem to solve is:

$$
\left\{\begin{array}{l}
\text { Trouver } \underline{\underline{a}}^{t} \in V, \text { vérifiant } \\
A\left(\underline{\underline{a}}, \underline{\underline{a}} \underline{\underline{a}}^{*}\right)=l(\underline{\underline{a}}) \text { pour tout } \underline{\underline{a}} \text { dans } V .
\end{array}\right.
$$

where $P=\left(L^{2}(\Omega)\right)^{d(d+1) / 2}$ and $V=\left\{\underline{\underline{K}} \in P, \nabla \underline{\underline{K}} \in P^{d}\right\}$ are classical functional spaces. The standard Galerkin technique will allows us to approach $a$ with an interpolation of order 1 in space, such that, in each element, the solution $\underline{\underline{a}}_{h}$ is written

$$
\underline{\underline{a}}_{h}=\sum_{i=1}^{\text {Nbnoeuds }} \underline{\underline{a}}_{j} N_{j}(x)
$$

where $N_{j}$ are polynomial functions of degree 1 . However, the resolution of the orientation equation using this approximation can be at the origin of wrong oscillations and a less accurate solution, since the problem is hyperbolic and can be convection dominant.

\section{Stabilisation}

If we follow a SUPG (Streamline Upwind Petrov Galerkin) stabilisation technique, the basic idea is to remove oscillations by perturbing the test function $\underline{a}$ by function $\underline{\underline{a}}^{*}$, defined as:

$\underline{\underline{\tilde{a}}}^{*}=\underline{\underline{a}}^{*}+\sigma \underline{v} \cdot \nabla \underline{\underline{a}}^{*}$

To ensure the consistency of the method, the coefficient $\sigma$ is computed over each element (with mesh size $h_{i}$ ) using the relation :

$\sigma_{i}=\frac{h_{i}}{2\left\|\underline{v}_{i}\right\|_{m o y}}$

$\left\|\underline{v}_{i}\right\|_{\text {moy }}$ being the average velocity on the element $\mathrm{i}$. One other stabilisation technique is the RFB (Residual Free Bubble) method that consists in enriching the finite space $V$ with a space $V_{B}$ denoted bubble function space. The total space is $V^{R F B}$ and is given by:

$V^{R F B}=V \oplus V_{B}$

Each element $\underline{a}_{h} \in V^{R F B}$ is written as the addition of a linear part $\underline{\underline{\alpha}}_{L} \in V$ and a bubble $\underline{\underline{a}}_{B} \in V_{B}$.

$\underline{\underline{a}}_{h}=\underline{\underline{a}}_{\mathrm{L}}+\underline{\underline{a}}_{B}$

The variational problem is :

$\left\{\begin{array}{l}\text { Chercher } \underline{\underline{a}}_{h}=\underline{\underline{a}}_{\mathrm{L}}+\underline{\underline{a}}_{B} \in V \oplus V_{B}, \text { telque pour tout } \underline{\underline{\underline{a}}}_{h}^{*}=\underline{\underline{a}}_{\mathrm{L}}^{*}+\underline{\underline{a}}_{B}^{*} \in V \oplus V_{B} \\ A\left(\underline{\underline{a}}+\underline{\underline{a}}_{B}, v\right)=l(v) \\ A\left(\underline{\underline{a}}+\underline{\underline{a}}_{B}, v\right)=l\left(v_{B}\right)\end{array}\right.$ (16)

\section{Discontinuous Galerkin}

The instabilities appearing with a standard Galerkin approximation can be also overcome using a discontinuous approximation. In Rem3D, a STDG (Space Time Discontinuous Galerkin) technique has been implemented. It allows a simultaneous resolution in space and in time by adding the additional variable time to the spatial computational domain. Thus, We redefine the operators $\tilde{v}$ and gradient $\tilde{\nabla}$ : 


$$
\tilde{v}=\left(\begin{array}{l}
v \\
1
\end{array}\right) ; \quad \tilde{\nabla}=\left(\begin{array}{c}
\nabla \\
\frac{\partial}{\partial t}
\end{array}\right)
$$

The orientation equation becomes :

$\underline{\tilde{v}} \cdot \underline{\tilde{\nabla}} \underline{\underline{a}}+B_{1} \cdot \underline{\underline{a}}+\underline{\underline{a}} \cdot B_{2}+F \cdot \underline{\underline{a}}=\frac{\underline{\underline{\underline{a}}}}{3 \theta}$

and is defined over the whole space-time domain $\tilde{\Omega}=\Omega \times[0, t]$. The time interval is structured $0=t_{0}<t_{1}<\ldots<t_{i}<t_{i+1}<\ldots<t_{n}=t$ and we define the temporal element by $I=] t_{i}, t_{i+1}[$. The space discretisation $\tilde{\Omega}$ consists in building a mesh of the space-time domain. The orientation tensor approximation is chosen discontinuous $\left(\mathrm{P}_{0}\right)$ in space and of order $n\left(\mathrm{P}_{\mathrm{n}}\right)$ in time. We will take as base functions on each element $\tilde{K}: \varphi_{\tilde{K}}=1_{\tilde{K}}(x, t)\left(t-t_{i}\right)^{q}$ with

$1_{\tilde{K}}(x, t)= \begin{cases}1 & \text { si } \tilde{x}=(x, t) \in \tilde{K} \\ 0 & \text { sinon }\end{cases}$

The orientation tensor $\underline{\underline{a}}$ and the test functions $\underline{\underline{a}}^{*}$ will be approached on each element by:

$$
\begin{aligned}
& \underline{\underline{a}}_{h}(x, t)_{\tilde{K}}=\sum_{p=0}^{n} \underline{\underline{a}}_{\tilde{K}}\left(t-t_{i}\right)^{p} ; \\
& \underline{\underline{a}}_{h}^{*}(x, t)_{\tilde{K}}=1_{\tilde{K}}(x, t)\left(t-t_{i}\right)^{r}
\end{aligned}
$$

\section{RESULTS AND DISCUSSION}

\section{Comparison Between Continuous and Discontinuous Galerkin}

We consider a cylindrical cavity, filled with a fluid that has an initial isotropic orientation (aii $=1 / 3$, aij $=0$ if $i \neq j$ ). The fluid flows through the cylinder, and we suppose it enters at the isotropic state.

After the transient period, we reach the steady state and obtain a typical skin-core structure (Figure 2). The component of the orientation tensor along the flow axis (azz) attains its maximum value at the boundary of the cylinder, while the transverse components (axx and ayy) have the opposite behavior. Furthermore, components (axz and ayz) vary over the thickness in a quasi-linear way. The distributions obtained at the steady state with the solvers P0 (Discontinuous Galerkin) and P1 (Continuous Galerkin) are given in Figure 2, where we can observe the same qualitative tendency. In what concerns the continuous solution we notice oscillations at the entrance, phenomena similar to a "shock», due to the imposed orientation condition. Even if it doesn't have any influence on the solution, periodic boundary conditions might overcome this problem.

\section{Continuous Galerkin Discontinuous Galerkin}

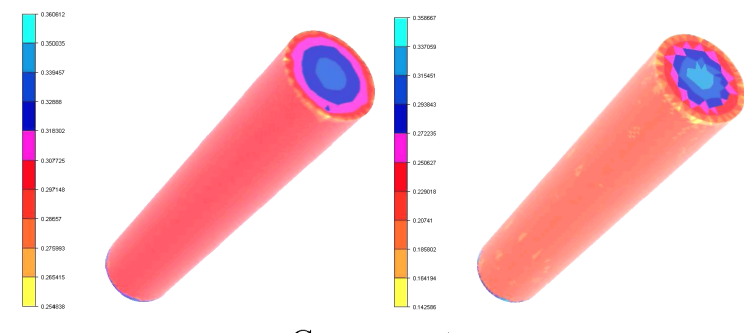

$$
\text { Component } a_{x x}
$$
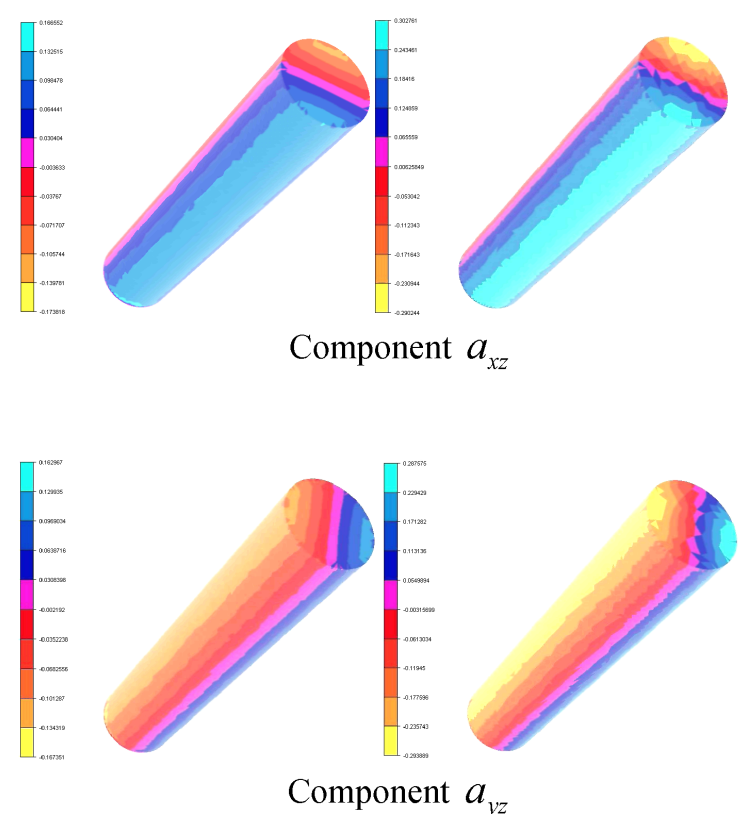

FIGURE 2. Orientation tensor components distribution once the steady-state is reached. 


\section{Results in an Injection Molding Test Case}

We consider the filling and post-filling of a volumic piece, with important thickness variations. The material used was a polyethylene (LDPE with 8 viscoelastic modes). The computation time for the whole thermomechanical cycle was of 1 day and 16 hours in 8 processors (each processor is a Pentium IV $2.8 \mathrm{GHz}$ and $1 \mathrm{~Gb}$ RAM, linked to the other processors by a Myrinet network.

Figure 3 shows the orientation distribution at different time steps, for the last mode (highest orientation time). We notice that orientations do not relax during the processus duration and are present at the ejection moment. A similar analysis can be done to the stretch evolution.

Figure 4 shows the first principal stress distribution in a section of the part at two different instants (filling and cooling, respectively). During filling, molecules located at the center are less oriented than the ones closer to the wall, due to the low shear rate, giving rise to lower flow induced-stresses and lower birefringence values. At the end of filling, the stresses tend to relax, since velocity reduces drastically. During packing, flow-induced stresses still exist, either frozen or due to material's motion and increase of the relaxation times. At the end cooling, stresses are frozen-in in regions of the part near the wall.

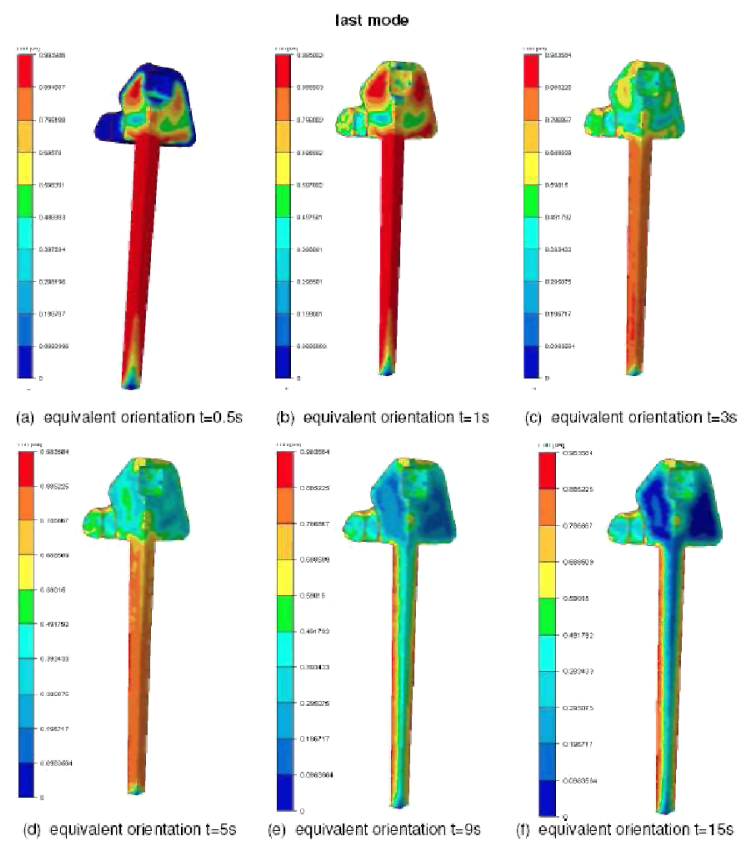

FIGURE 3. Equivalent orientation distribution for the last mode (the last picture represents the frozen-in orientation, at $\mathrm{t}=15 \mathrm{~s})$.
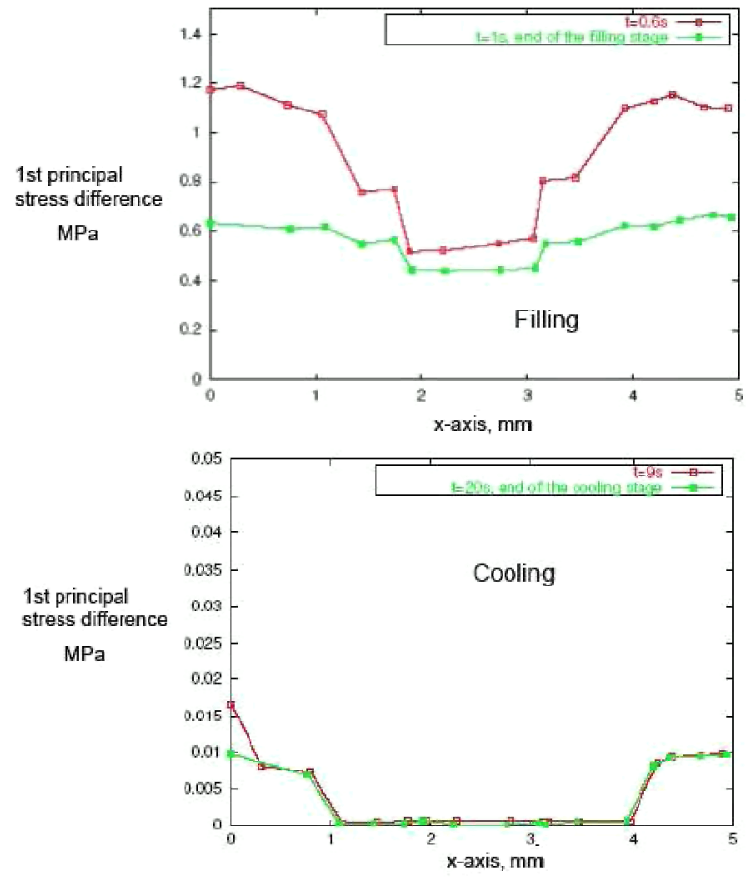

FIGURE 4. Equivalent orientation distribution for the last mode (the last picture represents the frozen-in orientation, at $\mathrm{t}=15 \mathrm{~s})$.

\section{CONCLUSIONS}

In this paper, we have shown how to compute the main descriptor of the anisotropic state of the material, the orientation tensor. The model described is valid both for fiber and for macromolecular chain orientation. Two resolution techniques have been described: the first one based on a continuous approximation of the tensor, the other on a discontinuous. A continuous approximation may lead to oscillations in the solution and requires stabilisation; a discontinuous approximation is more CPU consuming (since we have 5 times more elements than nodes in a 3D mesh) and less accurate. Utility in injection molding has been shown, through a complex example. The main perspectives of this work concern taking into account orientation in the mechanical properties.

\section{ACKNOWLEDGMENTS}

The authors thank the support of the Rem3D Consortium, which has as partners Arkema, Armines, Dow Chemicals, Schneider Electric, Snecma Propulsion Solide and Transvalor. 


\section{REFERENCES}

1. A. Isayev Polym. Eng. Science 23, 271 (1983).

2. G.G. Lipscomb, R. Keunigs, G. Marrucci and M.M. Denn in Proceedings of the IX International Congress on Rheology 2, 497 (1984).

3. A. Megally, "Etude et Modélisation de l'Orientation de Fibres dans les Thermoplastiques Renforcés", Ph.D. Thesis, Ecole des Mines de Paris, 2005.

4. L. Silva, "Viscoelastic Compressible Flow and Applications in 3D Injection Molding Simulation", Ph.D. Thesis, Ecole des Mines de Paris, 2005.

5. A. Flaman, "Built-Up and Relaxation of Moleculare Orientation in Injection Molding ", Ph.D. Thesis, Technische Universiteit Eindhoven, 1990.

6. S. Batkam, J. Bruchon and T. Coupez Int. J. Form. 7, 11 (2004). 